\title{
Supera Stent Implantation with Ultrasound Guidance for Complex Ostial Lesions of Superficial Femoral Artery by Retrograde Approach: The SUGAR Technique
}

\author{
Virender Singh Sheorain ${ }^{1}$ Rajiv Parakh ${ }^{1} \quad$ Tarun Grover $^{1} \quad$ Tapish Sahu $^{1} \quad$ Ashutosh Aher $^{1}$ \\ ${ }^{1}$ Department of Peripheral Vascular and Endovascular Sciences, \\ Address for correspondence Virender Singh Sheorain, MD, \\ Medanta - The Medicity, Gurgaon, Haryana, India \\ Department of Peripheral Vascular and Endovascular Sciences, \\ Medanta - The Medicity, Sector 38, Gurgaon 122001, Haryana, India \\ (e-mail: veeru40395@gmail.com).
}

J Clin Interv Radiol ISVIR 2021;5:167-171.

\author{
Abstract \\ Keywords \\ - plain old balloon \\ angioplasty \\ - vasculomimetic \\ - ostial lesion \\ - retrograde pedal \\ access \\ - superficial femoral \\ artery \\ - profunda femoral \\ artery \\ - stent
}

Purpose The aim of this study was to describe a modified technique for accurate deployment of the Supera stent under ultrasound (US) guidance for complex lesions involving superficial femoral artery (SFA) ostium without jailing profunda femoral artery (PFA) by retrograde approach.

Technique Retrograde pedal access was initially taken in all the cases. The stenotic or occlusive femoropopliteal lesions involving the SFA ostium were crossed retrogradely and additional contralateral common femoral artery (CFA) access was taken only if we failed to cross the lesion. In addition to fluoroscopy, extravascular US guidance was freely used whenever required throughout the procedure. After desired predilatation of the SFA lesion, US guidance (gray scale and color Doppler) was used to start deployment of the proximal few centimeters of Supera stent ensuring that the landing of first crown was exactly from the SFA ostium not extending into CFA or jailing the PFA ostium. The rest of the stent was deployed under fluoroscopic guidance. Retrograde pedal access site hemostasis was achieved by manual compression. The Supera stent implantation with Ultrasound Guidance for complex ostial lesions of superficial femoral Artery by Retrograde approach (SUGAR) technique was applied successfully in 35 patients (mean age $58.1 \pm 4.4$ years; 24 males) with critical limb ischemia via the distal pedal approach. No access site or remote complications were observed.

Conclusion The SUGAR technique has superior advantage of utilizing a simple tool like extravascular US in addition to fluoroscopy for retrograde Supera stent deployment by allowing very precise landing at SFA ostium, bypassing the need for multiple oblique projections, unwanted radiation exposure, and extra contrast dye overload. published online May 21, 2021
DOI https://doi.org/

$10.1055 / \mathrm{s}-0041-1728976$ ISSN 2457-0214
(C) 2021. Indian Society of Vascular and Interventional Radiology. This is an open access article published by Thieme under the terms of the Creative Commons Attribution-NonDerivative-NonCommercial-License, permitting copying and reproduction so long as the original work is given appropriate credit. Contents may not be used for commercial purposes, or adapted, remixed, transformed or built upon. (https://creativecommons.org/licenses/by-nc-nd/4.0/). Thieme Medical and Scientific Publishers Pvt. Ltd. A-12, 2nd Floor, Sector 2, Noida-201301 UP, India 


\section{Introduction}

The superficial femoral artery (SFA) per se is highly exposed to biomechanical external forces including compression, torsion, and elongation occurring during leg movement. ${ }^{1}$ If the occlusive lesion involves the ostium, it further adds to the complexity of treating SFA disease. Vascular lesions involving the SFA ostium often involve the common femoral bifurcation, are usually calcified, and are prone to recoil after plain old balloon angioplasty (POBA). ${ }^{2}$ The benefits of primary stenting over POBA for SFA ostial lesions after endovascular recanalization in the femoropopliteal segment is well proven by many studies. ${ }^{3-11}$

Most laser-cut self-expanding nitinol stents when placed in the SFA especially for ostial lesions are at risk of compression, kinking, and fractures, manifested as accelerated in-stent restenosis. ${ }^{12}$ The last decade has seen stents with new designs to meet the challenges in the SFA specially to reduce the incidence of stent fractures. Because of this evolution in stent design, many interventionists have started using vasculomimetic designs (Supera stent; Abbott Vascular, Santa Rosa, California, United States) over laser-cut nitinol stents for SFA lesions. These newer designs inhibit the creation of focal areas of increased wall stress, achieve greater radial strength, are more flexible, and are fracture resistant thus improving outcomes. ${ }^{5,10,13,14}$

The Supera stent is a new stent design. This stent has been proven to be fracture resistant in several registries ${ }^{15-17}$ and in U.S. Investigational Device Exemption trial. ${ }^{18}$ The stent is not a laser-cut nitinol tube but rather consists of six pairs of nitinol interwoven wires formed in a helical pattern to provide improved radial and longitudinal characteristics, answering the need for a fracture resistant stent with high flexibility and compression resistance. Whenever stenting becomes mandatory for complex femoropopliteal lesions involving the SFA ostium, we prefer Supera stent as this segment is exposed to high mechanical stress due to leg flexion, extension, and rotation.

While deploying the Supera stent in SFA by antegrade approach, one of the major challenges and limitations of such platform is the technical difficulty to finish landing of its last crown precisely at the SFA ostium without unwanted extension into common femoral artery (CFA) or "jailing" the profunda femoral artery (PFA). Hence for accurate landing of Supera stent, preferred approach is retrograde. A novel technical approach but solely under fluoroscopic guidance was proposed by Palena et al, known as precise retrograde Supera stenting of the SFA ostium (PRESTO technique) for complex femoropopliteal occlusions. The PRESTO technique was conceived and developed to guarantee accurate and safe deployment of the Supera stent in complex cases in which there is involvement of the SFA ostium. ${ }^{19}$

The superior advantage of ultrasound (US) guidance in addition to fluoroscopy while deploying Supera stent at femoral bifurcation by retrograde approach is the accurate landing of the first crown at the SFA ostium bypassing the need for multiple oblique projections, radiation exposure, and unwanted contrast dye overload. On occasions in spite of multiple oblique projections, we fail to separate SFA and PFA ostia. Each extra oblique angiogram leads to unwanted radiation exposure and contrast load. As most critical limb ischemia (CLI) patients are in pain, they tend to move thus leading to distortion of roadmap and difficulty in precise stent deployment. The above limitations of fluoroscopy can be easily overcome by use of US assistance done with linear array transducer focusing at the CFA bifurcation. Angulating the probe of US (gray scale and Duplex mode) helps to delineate SFA and PFA ostia with ease. With Supera stent implantation with Ultrasound Guidance for complex ostial lesions of superficial femoral Artery by Retrograde approach (SUGAR) technique, the operator can be more confident while landing the first crown of Supera from the SFA ostium.

To proficiently perform endovascular interventions with US guidance, operators must become familiar with the appearance of different devices on extravascular US. The appearance of all the laser-cut stents on US is nearly similar. However, Supera stent is not a laser-cut nitinol tube but rather made up of six pairs of nitinol interwoven wires in a helical pattern. It has a unique appearance on US and the operator can easily visualize its nose-cone, and the crowns or struts are seen as highly echogenic structure extending out of the delivery sheath with each stroke of pusher knob during its deployment. The apposition of the stent struts to the arterial wall is also well documented. Color Doppler mode when performed simultaneously at the time of deployment is very helpful to separate PFA ostium to prevent its inadvertent jailing.

\section{Technique}

The procedure steps were planned on preprocedure Duplex and/or computed tomography angiography findings. All the 35 patients who underwent endovascular procedure were on dual antiplatelets (DAPT) and statins. Bilateral groins and the entire affected lower extremity were prepared after placing patient in supine position on angiography table. Depending on the caliber and Duplex characteristics of the pedal vessel, either distal anterior tibial artery (ATA; 15 cases) or distal posterior tibial artery (PTA; 20 cases) was accessed. Micropuncture set with 21-gauge micropuncture needle (Cook Medical, Bloomington, Indiana, United States) was used to access the pedal arteries under US guidance. After administering 200 micrograms of nitroglycerin, the micropuncture sheath was exchanged for a 5/6-F Slender sheath (Terumo, Somerset, New Jersey, United States). An intravenous bolus of $70 \mathrm{U} / \mathrm{kg}$ of unfractionated heparin was administered. Retrograde tibial angiograms and extravascular US were performed as and when required negotiating the lesion. We worked with a 0.018 inch compatible system. The lesion was crossed using 0.018 CXI support catheter (Cook Medical, Bloomington, Indiana, United States) and 0.018 Command guidewire, and predilated using 0.018 Armada balloon catheter (Abbott Vascular, Santa Rosa, California, United States).

First attempt to recanalize the target vessel was always made via the retrograde pedal approach; however, if it failed then contralateral femoral access was obtained under US guidance. Once the SFA lesion was crossed, predilatation of 
the lesion was performed to the desired size. When stenting of the SFA ostium was mandatory, we preferred Supera stent over laser-cut self-expanding nitinol stents for the reasons discussed. As per instructions for use, vessel bed preparation was performed using balloon size $0.5 \mathrm{~mm}$ larger than the estimated Supera stent diameter. Its overdilatation allows the stent cells to coapt with the vessel wall and acquire its recommended diamond configuration.

The measured Supera stent was then advanced via the retrograde pedal access ( - Figs. 1 and $\mathbf{2}$ ). US of the CFA bifurcation was performed to delineate the SFA and PFA ostia (-Fig. 3). Under continuous live US, the nose-cone of Supera stent was parked just at the SFA ostium and deployment of Supera was performed very slowly till the first crown of the stent is seen extending out as bright echogenic structure from its delivery sheath. The stent crown/struct is seen as a bright echogenic structure ( - Fig. 4 ). If the first crown is noted advancing toward the CFA, it can be easily slightly pulled back into the SFA ostium. Simultaneously, color Doppler mode can be used to reconfirm the SFA and PFA ostia to prevent jailing of PFA ( - Fig. 5). Once the stent is deployed, beginning from the ostium to the proximal few centimeters in SFA, rest of the stent deployment can be performed under fluoroscopic guidance (-Fig. 6). Post stenting and remodeling, a final angiogram was performed using a 4 F CXI support catheter parked in CFA/external iliac artery.

In addition to initial retrograde pedal approach, additional contralateral femoral access was required to either achieve reentry or to deploy the second overlapping Supera stent (for longer length lesions) for precise landing into distal SFA-popliteal segment after initial retrograde placement of the proximal Supera stent for SFA ostial lesion using SUGAR technique. Out of the 35 Supera stents placed for SFA ostial lesions, 20 were placed via PTA access and 15 via ATA access. If required, patients who underwent femoropopliteal stenting also underwent concomitant or staged tibial and/or pedal intervention to increase foot perfusion. Accurate landing of the Supera stent was achieved in all the cases precisely from the SFA ostium utilizing the SUGAR technique. Adequate hemostasis was achieved at the retrograde access sites in all cases. No access site or distant complications were observed. After the procedure, DAPT was continued for at least 6 months. All the patients in our series had CLI with Rutherford category $5 / 6$ ischemia, moderate $(n=20)$ or severely $(n=15)$ calcified chronic total occlusions (CTOs; mean length: $21.2 \pm 11.3 \mathrm{~cm}$, range: $9.9-32.5)$ in the SFA ( $n=28$ ) or femoropopliteal segment $(n=7)$, and at least one or two patients had tibial runoff vessels. The 6 -month follow-up in all patients disclosed no stent fracture or occlusion.

\section{Discussion}

CFA is still considered a no-stent zone. Multiple potential negative effects of stenting CFA include possibility of plaque shift compromising PFA flow, limiting future surgical options in cases of stent failure, and precluding access for future interventions. Importance of the PFA cannot be ignored because of its role in collateral circulation to the calf in patients with SFA occlusion and it needs utmost respect.

Several different endovascular therapies are available to treat the complex femoropopliteal lesions involving SFA ostium, such as POBA, atherectomy, drug-coated balloons, intravascular lithotripsy, and laser-cut self-expanding nitinol and drug-eluting stents (DES).

Available self-expanding nitinol stents and DES have improved results in the femoropopliteal segment; however, lesion morphology remains the most important predictor of long-term outcomes. Long, calcified CTOs represent the most complex lesion subtype and are associated with the highest incidence of stent fractures. ${ }^{12}$

Moreover, the constant exposure to biodynamic forces such as compression, torsion, flexion, extension, or rotation in the femoropopliteal segment exerts significant stress on metallic endoprostheses ${ }^{5}$ leading to compression, kinking, and fractures with an inflammatory restenotic response. Newer-generation stents incorporating biomimetic features into their design, like Supera stent, have shown improved outcomes when compared with POBA,, ${ }^{511-13}$ with the added benefit of freedom from fracture. However, given the eccentricities related to the deployment mechanism of the Supera stent, the release of the distal most or the first crown of the stent is always perfectly predictable, which is not necessarily


Fig. 1 (A) Distal anterior tibial artery access and (B) distal posterior tibial artery access with 5/6 slender sheath. 




Fig. 2 Retrograde advancement of Supera stent through the distal posterior tibial artery access.
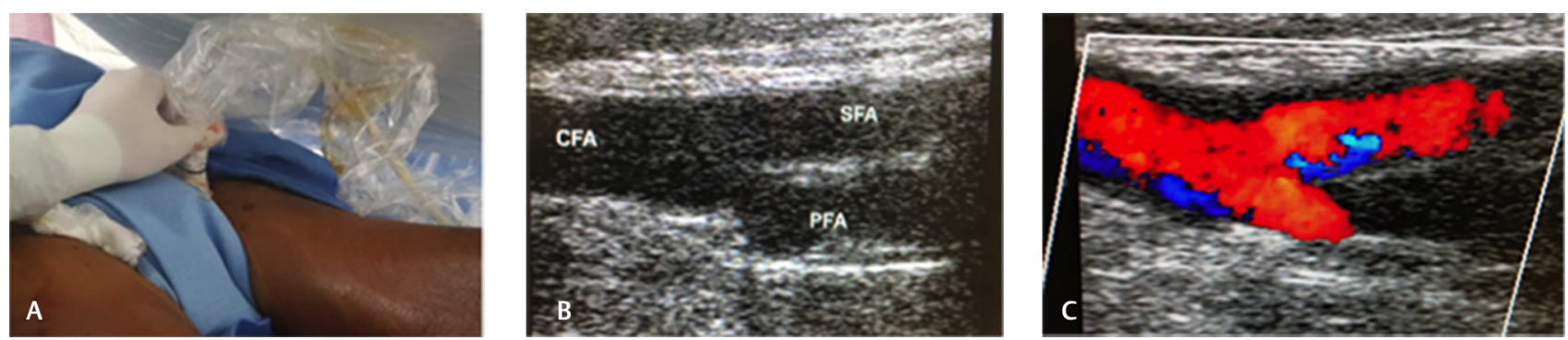

Fig. 3 Ultrasound scan of the groin: (A) linear probe, (B) gray-scale image of the common femoral artery (CFA) bifurcation, and (C) color Doppler image of the CFA bifurcation. SFA, superficial femoral artery.



Fig. 4 Supera stent as seen on gray-scale ultrasound with linear transducer.

true for the proximal most or the last crown to be deployed. Most operators tend to avoid its use in antegrade fashion and prefer laser-cut self-expanding nitinol stent to achieve perfect landing at the SFA ostium. The retrograde deployment of the Supera stent allows the operator to precisely and safely release the first crown of the stent right from the SFA ostium. Vasculomimetic platform should be one of the preferred choices in challenging cases when stent is mandatory in this anatomical location.

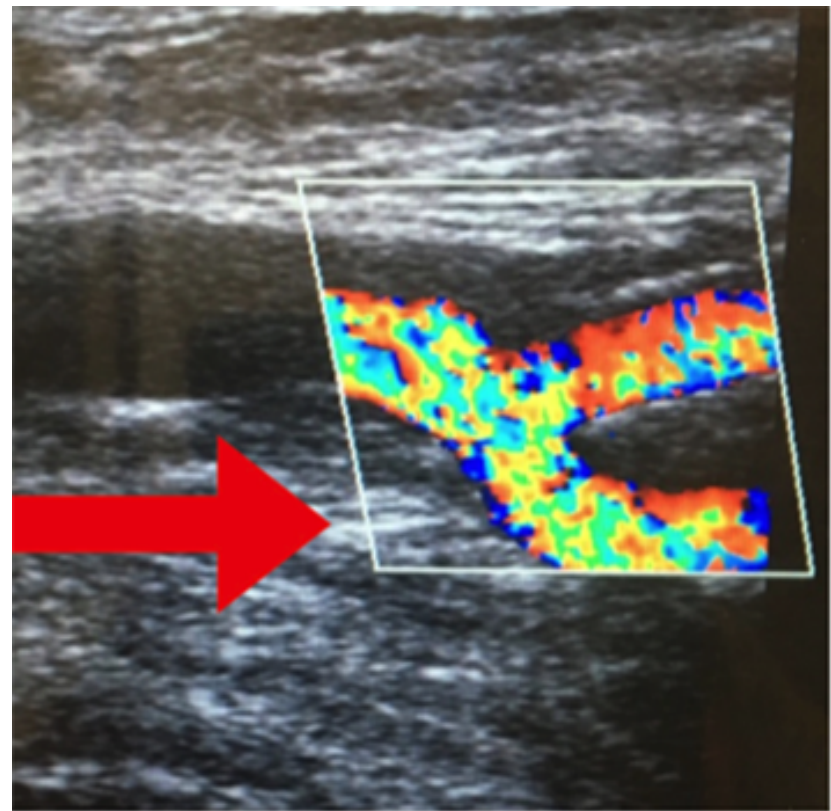

Fig. 5 Color Doppler image showing accurately deployed proximal Supera stent exactly from superficial femoral artery ostium without "jailing" profunda femoral artery.

To the best of our knowledge, we could not find any published usage of extravascular US specifically to assist retrograde deployment of Supera stent platform from the SFA ostium. 




Fig. 6 Fluoroscopic image showing partially deployed proximal Supera stent.

\section{Conclusion}

In selected group of patients, the SUGAR technique has a superior advantage over fluoroscopy alone for retrograde Supera stent deployment by allowing precise landing at SFA ostium, bypassing the need for multiple oblique angiograms, unwanted radiation exposure, and extra contrast dye overload. Further experiences with larger population are required.

\section{Conflict of Interest}

None declared.

\section{References}

1 Scheinert D, Scheinert S, Sax J, et al. Prevalence and clinical impact of stent fractures after femoropopliteal stenting. J Am Coll Cardiol 2005;45(2):312-315

2 Yamawaki M, Hirano K, Nakano M, et al. Deployment of self-expandable stents for complex proximal superficial femoral artery lesions involving the femoral bifurcation with or without jailed deep femoral artery. Catheter Cardiovasc Interv 2013;81(6):1031-1041

3 Jaff MR, White CJ, Hiatt WR, et al. TASC Steering Committee. An update on methods for revascularization and expansion of the TASC lesion classification to include below-theknee arteries: a supplement to the Inter-Society Consensus for the Management of Peripheral Arterial Disease (TASC II) J Endovasc Ther 2015;22(5):663-677

4 Duda SH, Bosiers M, Lammer J, et al. Drug-eluting and bare nitinol stents for the treatment of atherosclerotic lesions in the superficial femoral artery: long-term results from the SIROCCO trial. J Endovasc Ther 2006;13(6):701-710
5 Krankenberg H, Schlüter M, Steinkamp HJ, et al. Nitinol stent implantation versus percutaneous transluminal angioplasty in superficial femoral artery lesions up to $10 \mathrm{~cm}$ in length: the Femoral Artery Stenting Trial (FAST). Circulation 2007;116(3):285-292

6 Zeller T, Tiefenbacher C, Steinkamp HJ, et al. Nitinol stent implantation in TASC A and B superficial femoral artery lesions: the Femoral Artery Conformexx Trial (FACT) J Endovasc Ther 2008;15(4):390-398

7 Schillinger M, Sabeti S, Loewe C, et al. Balloon angioplasty versus implantation of nitinol stents in the superficial femoral artery. N Engl J Med 2006;354(18):1879-1888

8 Dake MD, Scheinert D, Tepe G, et al. Zilver PTX Single-Arm Study Investigators. Nitinol stents with polymer-free paclitaxel coating for lesions in the superficial femoral and popliteal arteries above the knee: twelve-month safety and effectiveness results from the Zilver PTX single-arm clinical study. J Endovasc Ther 2011;18(5):613-623

9 Laird JR, Katzen BT, Scheinert D, et al. RESILIENT Investigators. Nitinol stent implantation vs. balloon angioplasty for lesions in the superficial femoral and proximal popliteal arteries of patients with claudication: three-year follow-up from the RESILIENT randomized trial. J Endovasc Ther 2012;19(1):1-9

10 Bosiers M, Torsello G, Gissler HM, et al. Nitinol stent implantation in long superficial femoral artery lesions: 12-month results of the DURABILITY I study. J Endovasc Ther 2009;16(3):261-269

11 Sabeti S, Mlekusch W, Amighi J, Minar E, Schillinger M. Primary patency of long-segment self-expanding nitinol stents in the femoropopliteal arteries. J Endovasc Ther 2005;12(1):6-12

12 Laird JR. Limitations of percutaneous transluminal angioplasty and stenting for the treatment of disease of the superficial femoral and popliteal arteries. J Endovasc Ther 2006;13 (Suppl 2) :II30-II40

13 Laird JR, Katzen BT, Scheinert D, et al. RESILIENT Investigators. Nitinol stent implantation versus balloon angioplasty for lesions in the superficial femoral artery and proximal popliteal artery: twelve-month results from the RESILIENT randomized trial. Circ Cardiovasc Interv 2010;3(3):267-276

14 Dake MD, Ansel GM, Jaff MR, et al. Zilver PTX Investigators. Paclitaxel-eluting stents show superiority to balloon angioplasty and bare metal stents in femoropopliteal disease: twelve-month Zilver PTX randomized study results. Circ Cardiovasc Interv 2011;4(5):495-504

15 Werner M, Paetzold A, Banning-Eichenseer U, et al. Treatment of complex atherosclerotic femoropopliteal artery disease with a self-expanding interwoven nitinol stent: midterm results from the Leipzig SUPERA 500 registry. EuroIntervention 2014;10(7):861-868

16 Scheinert D, Grummt L, Piorkowski M, et al. A novel self-expanding interwoven nitinol stent for complex femoropopliteal lesions: 24-month results of the SUPERA SFA registry. J Endovasc Ther 2011;18(6):745-752

17 Scheinert D, Werner M, Scheinert S, et al. Treatment of complex atherosclerotic popliteal artery disease with a new self-expanding interwoven nitinol stent: 12-month results of the Leipzig SUPERA popliteal artery stent registry. JACC Cardiovasc Interv 2013;6(1):65-71

18 Piorkowski M, Freitas B, Schmidt A, et al. The use of the GORE $®$ TIGRIS $®$ Vascular Stent with dual component design in the superficial femoral and popliteal arteries at 6 months. J Cardiovasc Surg (Torino) 2013;54(4):447-453

19 Palena LM, Diaz-Sandoval LJ, Raja LM, Morelli L, Manzi M. Precise retrograde Supera stenting of the ostium (PRESTO) of the superficial femoral artery for complex femoropopliteal occlusions: the PRESTO technique. J Endovasc Ther 2018;25(5):588-591 